\title{
Levantamento bibliométrico dos artigos publicados na Revista da ABENO no período entre 2001 e 2019
}

\author{
Allan Vinícius Martins de Barros*; Vitória Helena Sales do Nascimento**; Caio César Gonçalves \\ Silva***; Bruna Bezerra do Amaral****; Renata de Oliveira Cartaxo*****; Pedro Henrique Sette-de- \\ Souza $* * * * *$
}

* Residente em Cirurgia e Traumatologia Buco-Maxilo-Facial, Hospital Universitário Oswaldo Cruz da Universidade de Pernambuco

** Graduanda em Odontologia, Faculdade de Odontologia de Pernambuco

*** Mestrando, Programa de Pós-Graduação em Odontologia, área de concentração em Cirurgia e Traumatologia BucoMaxilo-Facial, Faculdade de Odontologia de Pernambuco

**** Cirurgiã-dentista, graduada pela Universidade de Pernambuco, Campus Arcoverde

***** Docente, Curso de Odontologia, Universidade de Pernambuco, Campus de Arcoverde

Recebido em 23/04/2020. Aprovado em 21/06/2020.

\begin{abstract}
RESUMO
O presente estudo objetivou traçar, por meio de análise bibliométrica, o perfil de produção científica da Revista da ABENO. A estratégia de busca foi conduzida por dois pesquisadores independentes no sítio web da revista, no qual foram analisados todos os artigos completos publicados no período entre 2001 e 2019. Mediante a leitura dos resumos, os trabalhos foram caracterizados de acordo com a categoria de estudo, a temática abordada, ano e volume de publicação, além de dados das IES às quais os autores do artigo eram vinculados, tais como natureza jurídica e localidade geográfica. Foram identificados 486 artigos publicados no período estudado. Majoritariamente, os artigos apresentaram delineamento metodológico de pesquisa quantitativa (46,3\%), cujas temáticas abordadas estavam relacionadas às áreas de "Ensino/Aprendizagem em Odontologia" $(49,8 \%)$ "Atividades de Estágio, Extensão e PET-Saúde" (15,2\%) e "Diretrizes Curriculares Nacionais" (10,1\%). Os estudos foram desenvolvidos em sua maior parte em instituições públicas (70\%), com predominância de localização na região Sudeste $(41,4 \%)$. Em conclusão, verifica-se que, ao longo de quase duas décadas, a Revista da ABENO apresentou volume crescente de publicações, com participação expressiva de IES, em especial das públicas, e com concentração desta produção na região Sudeste, embora com tendência a um aumento na participação das demais regiões geográficas.
\end{abstract}

Descritores: Bibliometria. Odontologia. Educação em Odontologia. 


\section{INTRODUÇÃO}

Levantamentos bibliométricos caracterizamse como estudos que avaliam atividades de produção e desempenho científico por meio de métodos estatísticos. O termo bibliometria foi introduzido por Otlet em 1934 para descrever o estudo dos padrões de comportamento da literatura e da comunicação, originalmente conhecido como "bibliografia estatística"1. Com o advento das bases de dados online e dos softwares de análise bibliométrica houve uma considerável popularização deste tipo de estudo aplicado às mais diversas áreas do conhecimento ${ }^{2}$.

A bibliometria é uma importante ferramenta na avaliação dos padrões de produção e disseminação do conhecimento ${ }^{1}$. Os métodos bibliométricos permitem o mapeamento de temas, autores e instituições atrelados à produção científica em um dado campo da ciência, bem como possibilitam a construção de indicadores capazes de avaliar os padrões e tendências relacionados a estes parâmetros, fornecendo informações úteis no direcionamento da investigação científica ${ }^{2,3}$.

Idealizada em 1956 durante uma reunião de entidades como a Coordenação de Aperfeiçoamento de Pessoal de Nível Superior (CAPES), o Serviço Especializado de Saúde Pública (SESP) e o Ponto IV, a Associação Brasileira de Ensino Odontológico (ABENO), inicialmente conhecida como Associação Brasileira de Estabelecimentos de Ensino Odontológico (ABEEO), foi fundada com o objetivo de traçar soluções para problemas do ensino odontológico brasileiro. Desde então, a ABENO vem incentivando e apoiando as mudanças no contexto educacional nos cursos de Odontologia das Instituições de Ensino Superior (IES) do Brasil ${ }^{4}$.

Dentre outras competências, a ABENO realiza reuniões científicas nacionais de caráter anual com o objetivo de compartilhar experiências de mudanças relativas à educação e ao ensino odontológico, como as tendências de produção acadêmica no âmbito do ensino, pesquisa e extensão em Odontologia, a implantação das metodologias ativas de ensino e aprendizagem e as percepções de discentes e docentes a respeito destes processos ${ }^{4}$.

Em 2001 a ABENO lançou o seu periódico, conhecido como Revista da ABENO, cuja missão principal é contribuir para a obtenção de indicadores de qualidade do ensino odontológico, com vistas a assegurar o contínuo progresso da formação profissional, produzindo benefícios diretamente voltados à coletividade. Desde então, a Revista da ABENO apresenta publicações que abordam temáticas relevantes para o ensino na área odontológica, recebendo trabalhos advindos de diversas IES do país ${ }^{4}$.

Assim, a Revista da ABENO é uma publicação oficial da Associação Brasileira de Ensino Odontológico que tem como princípio contribuir para a obtenção de indicadores de qualidade do ensino. Foi criada em 2001 iniciando com uma edição impressa anual e a partir de 2005 passou a publicar edições semestrais, tendo distribuição impressa gratuita aos sócios e às instituições de ensino superior e, em 2011, iniciou sua participação na iniciativa Rev@Odonto, um portal de revistas brasileiras de Odontologia. A contar do ano de 2015, as edições passaram a ser publicadas trimestralmente em meio eletrônico, por meio do Sistema Eletrônico de Editoração de Revistas (SEER), com o objetivo principal de reduzir custos, aumentar a visibilidade do conteúdo publicado e acelerar a revisão por pares.

Neste contexto, o padrão de produção e divulgação científica da Revista da ABENO possibilita identificar as tendências e o crescimento do conhecimento no campo do Ensino Odontológico, subsidiando o desenvolvimento de estratégias institucionais para o seu fortalecimento. Portanto, o presente estudo objetivou investigar, 
por meio de análise bibliométrica, o perfil de produção científica da Revista da ABENO no período entre 2001 e 2019.

\section{METODOLOGIA}

Esta pesquisa bibliométrica caracteriza-se como um estudo transversal com abordagem quantitativa. Foram analisados os artigos completos publicados na Revista da ABENO no período entre 2001 e 2019, que se encontram disponíveis para acesso livre e imediato no sítio web https://revabeno.emnuvens.com.br/revabeno.

Os artigos foram categorizados mediante leitura dos títulos e resumos. Nos casos em que não foi possível identificar alguma destas características por meio da leitura de título e resumo, o artigo foi lido na integra. Para a categorização dos artigos, foram consideradas as variáveis de desfecho: categoria do estudo e tema abordado, e as variáveis independentes: ano de publicação, natureza jurídica e localização de todas as IES às quais os autores do artigo eram vinculados.

A categoria do estudo foi definida com base no desenho metodológico adotado, sendo os artigos classificados em: pesquisa quantitativa, considerando as subcategorias de análise documental, estudos de intervenção, estudos prospectivos, estudos retrospectivos e estudos transversais; pesquisa qualitativa; pesquisa com metodologia híbrida, qualitativa e quantitativa; revisão da literatura, incluídas nesta categoria as revisões narrativas, integrativas e sistemáticas; e relato de experiência. O tema abordado foi identificado por meio da leitura dos componentes textuais do artigo e, de acordo o objeto de estudo, foram classificados em: Ensino/Aprendizagem em Odontologia; Atividades de Estágio, Extensão e PET-Saúde; Atividades de Pesquisa e Iniciação Científica; Diretrizes Curriculares Nacionais; Teleodontologia e Telessaúde; Mundo do Trabalho; e Epidemiologia e Serviços de Saúde.
Nos casos em que não foi possível categorizar o artigo em alguma destas temáticas, o mesmo foi enquadrado na categoria "Outros Temas". A natureza jurídica das IES foi categorizada em pública ou privada, sendo os artigos vinculados a IES com naturezas jurídicas distintas categorizados como parceria entre IES públicas e privadas. A localização das IES foi categorizada por meio da região geográfica, quando se tratava de IES brasileiras, e como internacional, quando se localizava em outro país. Nos casos em que não havia registro da filiação dos autores a qualquer instituição de ensino, estes foram considerados como sem vinculação a IES.

O processo de construção do banco de dados foi realizado por dois pesquisadores distintos (A.V.M.B. e B.B.A), que coletaram os mesmos dados de forma independente, construindo, desta forma, dois bancos de dados separados, verificados por meio de consistência seguidos de avaliação comparativa realizada por um terceiro pesquisador (P.H.S.S). Nos casos de divergência entre os dois avaliadores, o artigo era submetido a uma nova análise e o mesmo era categorizado de acordo com um consenso entre os três pesquisadores.

Os dados foram tabulados com o auxílio do Software SPSS $®$ (Statistical Package for Social Sciences) v. 20.0 e, ao fim desta etapa, os dados foram tratados por meio de análise descritiva univariada. Foi realizada, ainda, a análise bivariada descritiva da distribuição dos artigos por ano de publicação e localização das IES.

\section{RESULTADOS}

Foram identificados 486 artigos publicados na Revista da ABENO no período entre 2001 e 2019. Referente ao ano de publicação, verificou-se maior quantidade de trabalhos publicados em 2018, contabilizando 76 artigos (15,6\%), seguido pelos anos $2019(n=57)$ e $2017(n=52)$, conforme tabela 1 e gráfico 1.

Ainda é possível destacar na tabela 1 a 
distribuição das publicações de acordo com as regiões geográficas do país. A região Sudeste representou 41,3\% das publicações nacionais na Revista da ABENO, com 201 trabalhos, enquanto a região Norte apresentou o menor percentual
$(2,8 \%)$. As publicações vinculadas a IES internacionais representaram 10 artigos, equivalentes a $2,1 \%$ do total analisado, sendo oito provenientes de parceria entre IES brasileiras e internacionais.

Tabela 1. Distribuição dos artigos publicados na Revista da ABENO entre os anos 2001 e 2019 de acordo com o ano de publicação e a região geográfica das IES vinculadas

\begin{tabular}{|c|c|c|c|c|c|c|c|c|c|c|c|c|c|c|c|c|}
\hline \multirow{3}{*}{ Ano } & \multicolumn{16}{|c|}{ Região geográfica da instituição de vinculação } \\
\hline & \multicolumn{2}{|c|}{ Norte } & \multicolumn{2}{|c|}{ Nordeste } & \multicolumn{2}{|c|}{ Centro-Oeste } & \multicolumn{2}{|c|}{ Sudeste } & \multicolumn{2}{|c|}{ Sul } & \multicolumn{2}{|c|}{ Internacional } & \multicolumn{2}{|c|}{ Sem IES } & \multicolumn{2}{|c|}{ Total } \\
\hline & $\mathrm{n}$ & $\%$ & $\mathrm{n}$ & $\%$ & $\mathrm{n}$ & $\%$ & $\mathrm{n}$ & $\%$ & $\mathrm{n}$ & $\%$ & $\mathrm{n}$ & $\%$ & $\mathrm{n}$ & $\%$ & $\mathrm{n}$ & $\%$ \\
\hline 2001 & 00 & 0,0 & 00 & 0,0 & 02 & 33,3 & 04 & 66,6 & 00 & 0,0 & 00 & 0,0 & 01 & 16,6 & 06 & 100,0 \\
\hline 2002 & 00 & 0,0 & 00 & 0,0 & 01 & 20,0 & 03 & 60,0 & 01 & 20,0 & 00 & 0,0 & 01 & 20,0 & 05 & 100,0 \\
\hline 2003 & 00 & 0,0 & 00 & 0,0 & 01 & 6,6 & 06 & 40,0 & 03 & 20,0 & 01 & 6,6 & 04 & 26,6 & 15 & 100,0 \\
\hline 2004 & 00 & 0,0 & 01 & 10,0 & 02 & 20,0 & 04 & 40,0 & 03 & 30,0 & 00 & 0,0 & 00 & 0,0 & 10 & 100,0 \\
\hline 2005 & 00 & 0,0 & 02 & 10,0 & 03 & 15,0 & 12 & 60,0 & 02 & 10,0 & 00 & 0,0 & 01 & 5,0 & 20 & 100,0 \\
\hline 2006 & 00 & 0,0 & 03 & 13,6 & 00 & 0,0 & 12 & 54,5 & 06 & 27,2 & 00 & 0,0 & 02 & 9,0 & 22 & 100,0 \\
\hline 2007 & 00 & 0,0 & 02 & 10,5 & 02 & 10,5 & 12 & 63,1 & 03 & 15,7 & 00 & 0,0 & 00 & 0,0 & 19 & 100,0 \\
\hline 2008 & 00 & 0,0 & 03 & 18,7 & 02 & 12,5 & 10 & 62,5 & 02 & 12,5 & 00 & 0,0 & 00 & 0,0 & 16 & 100,0 \\
\hline 2009 & 01 & 7,1 & 01 & 7,1 & 00 & 0,0 & 06 & 42,8 & 07 & 50,0 & 00 & 0,0 & 00 & 0,0 & 14 & 100,0 \\
\hline 2010 & 00 & 0,0 & 04 & 21,0 & 01 & 5,2 & 08 & 42,1 & 05 & 26,3 & 00 & 0,0 & 01 & 5,2 & 19 & 100,0 \\
\hline 2011 & 00 & 0,0 & 03 & 12,5 & 01 & 4,1 & 11 & 45,8 & 10 & 41,6 & 00 & 0,0 & 00 & 0,0 & 24 & 100,0 \\
\hline 2012 & 02 & 14,2 & 03 & 21,4 & 00 & 0,0 & 03 & 21,4 & 06 & 42,8 & 00 & 0,0 & 00 & 0,0 & 14 & 100,0 \\
\hline 2013 & 00 & 0,0 & 02 & 18,1 & 00 & 0,0 & 06 & 54,5 & 04 & 36,3 & 00 & 0,0 & 00 & 0,0 & 11 & 100,0 \\
\hline 2014 & 00 & 0,0 & 02 & 18,1 & 00 & 0,0 & 04 & 36,3 & 05 & 45,4 & 00 & 0,0 & 00 & 0,0 & 11 & 100,0 \\
\hline 2015 & 03 & 6,2 & 14 & 29,1 & 00 & 0,0 & 16 & 33,3 & 19 & 39,5 & 02 & 4,1 & 01 & 2,0 & 48 & 100,0 \\
\hline 2016 & 02 & 4,2 & 15 & 31,9 & 02 & 4,2 & 18 & 38,2 & 21 & 44,6 & 01 & 2,1 & 00 & 0,0 & 47 & 100,0 \\
\hline 2017 & 02 & 3,8 & 06 & 11,5 & 08 & 15,3 & 21 & 40,3 & 25 & 48,0 & 01 & 1,9 & 00 & 0,0 & 52 & 100,0 \\
\hline 2018 & 01 & 1,3 & 23 & 30,2 & 13 & 17,1 & 26 & 34,2 & 25 & 32,8 & 03 & 3,9 & 00 & 0,0 & 76 & 100,0 \\
\hline 2019 & 03 & 5,2 & 13 & 22,8 & 08 & 14,0 & 19 & 33,3 & 20 & 35,0 & 02 & 3,5 & 00 & 0,0 & 57 & 100,0 \\
\hline Total & 14 & 2,8 & 97 & 19,9 & 46 & 9,4 & 201 & 41,3 & 167 & 34,3 & 10 & 2,0 & 11 & 2,2 & 486 & 100,0 \\
\hline
\end{tabular}




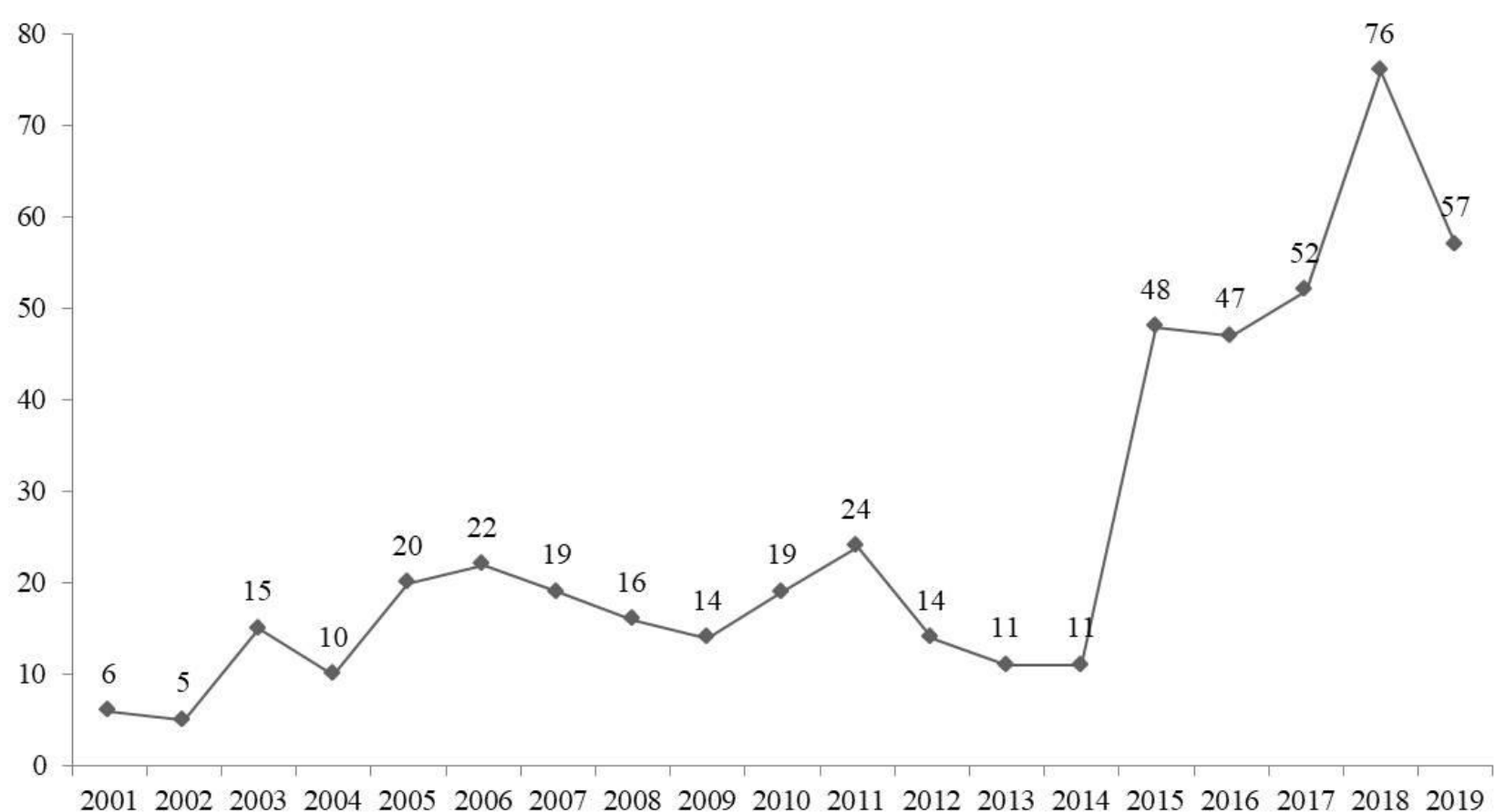

Gráfico 1. Série histórica dos artigos publicados na Revista da ABENO entre os anos 2001 e 2019

Em relação à variável "categoria do estudo", dos 486 trabalhos analisados, 225 (46,3\%) tratavam de pesquisa quantitativa, sendo os estudos de corte transversal os mais frequentes $(n=91$; $39,3 \%)$, enquanto $121(24,9 \%)$ eram de relatos de experiência e $71(14,6 \%)$ eram revisões da literatura (tabela 2).

A distribuição dos trabalhos de acordo com a natureza jurídica de administração da IES às quais os autores eram vinculados (pública, privada ou parceria entre os dois tipos), observada na tabela 2 , ilustra que a maioria dos artigos publicados é proveniente de instituições públicas, representando $340(70 \%)$ do total. Onze (2,3\%) artigos não tiveram autores vinculados a IES.

A tabela 2 ainda ilustra a distribuição dos trabalhos de acordo com os temas abordados. O maior número de publicações concentrou-se na área de "Ensino/Aprendizagem em Odontologia" $(49,8 \%)$, seguido por trabalhos que abordaram "Atividades de Estágio, Extensão e PET-Saúde"
(15,2\%) e "Diretrizes Curriculares Nacionais" $(10,1 \%)$. Trabalhos abordando outras temáticas menos frequentes e, portanto, não listadas corresponderam a 2,3\% do total de arquivos e, dentre estes, os temas relacionados à infraestrutura dos cursos de Odontologia e banco de dentes foram os mais abordados.

\section{DISCUSSÃO}

A bibliometria é uma ferramenta básica para a avaliação da ciência e estudos que utilizam métodos quantitativos para medição da produção científica estão em expansão em diversos países ${ }^{5}$. Dentre algumas aplicações das técnicas bibliométricas, destacam-se a possibilidade de determinar, entre outros aspectos, indicadores de tendência e crescimento em qualquer campo do conhecimento, com base na variação cronológica do número de trabalhos publicados e de sua distribuição geográfica, bem como da produtividade de autores e IES e da colaboração 
Tabela 2. Categorização dos artigos publicados na revista da ABENO entre os anos 2001 e 2019 segundo Natureza Jurídica da IES, Tipo de Estudo e Tema Abordado

\begin{tabular}{|c|c|c|}
\hline \multirow[b]{2}{*}{ Natureza jurídica das IES } & \multicolumn{2}{|c|}{ Número de Artigos } \\
\hline & $\mathbf{n}$ & $\%$ \\
\hline Públicas & 340 & 70.0 \\
\hline Privadas & 91 & 18.7 \\
\hline Parceria entre públicas e privadas & 44 & 9.0 \\
\hline Sem vinculação a IES & 11 & 2.3 \\
\hline Tipo de Estudo & $\mathbf{n}$ & $\%$ \\
\hline Pesquisa quantitativa & 225 & 46.3 \\
\hline Análise Documental & 14 & 2.9 \\
\hline Estudo de intervenção & 02 & 0.4 \\
\hline Estudo prospectivo & 13 & 2.7 \\
\hline Estudo retrospectivo & 05 & 1.0 \\
\hline Estudo transversal & 191 & 39.3 \\
\hline Pesquisa qualitativa & 49 & 10.1 \\
\hline Pesquisa qualitativa e quantitativa & 20 & 4.1 \\
\hline Relatos de Experiência & 121 & 24.9 \\
\hline Revisão da Literatura & 71 & 14.6 \\
\hline Tema abordado & $\mathbf{n}$ & $\%$ \\
\hline Ensino/Aprendizagem em Odontologia & 242 & 49.8 \\
\hline Atividades de Estágio, Extensão e PET-Saúde & 74 & 15.2 \\
\hline Atividades de Pesquisa e Iniciação Científica & 19 & 3.9 \\
\hline Diretrizes Curriculares Nacionais & 49 & 10.1 \\
\hline Epidemiologia e Serviços de Saúde & 42 & 8.6 \\
\hline Mundo do Trabalho & 39 & 8.0 \\
\hline Teleodontologia e Telessaúde & 10 & 2.1 \\
\hline Outros Temas & 11 & 2.3 \\
\hline Total & 486 & 100.0 \\
\hline
\end{tabular}

entre pesquisadores e/ou instituições, medidas pelo quantitativo de suas publicações ${ }^{6}$.

Deste modo, o levantamento bibliométrico dos artigos publicados na Revista da ABENO permite a caracterização do seu padrão de produção e divulgação científica e uma análise dos principais temas abordados e tendências na produção do conhecimento referente ao Ensino Odontológico e 
do perfil de produtividade de autores e IES. Este é o primeiro estudo de análise bibliométrica deste periódico científico, em seus 20 anos de publicações.

Atualmente, a Revista da ABENO publica em média 12 artigos por edição, além dos suplementos, classificados a partir do sistema Qualis em B3, redigidos em português/inglês ou espanhol $^{7}$. A evolução quanto ao quantitativo de publicações pode ser notada nesta série histórica, que evidencia o aumento gradual do número de artigos publicados por ano.

Hilu e Gisi (2011) ${ }^{8}$ destacam que a produção cientifica brasileira ainda é polarizada e concentrase em poucas IES, sendo mais de $90 \%$ das publicações geradas em universidades públicas, com uma baixa participação das IES privadas neste processo. Neste sentido, as publicações da Revista da ABENO seguem este mesmo padrão, como observado no presente estudo. $\mathrm{O}$ alto índice de publicações vinculadas a instituições públicas pode estar relacionado com o maior recebimento de recursos por meio de agências de fomento, pelo grande número de pesquisadores que congregam e por maior tradição na realização de pesquisas, principalmente pelas universidades vinculadas aos programas de pós-graduação stricto sensu ${ }^{9}$. Baseado nesses dados, os resultados observados apontam para a importância do investimento em universidades e institutos de pesquisa públicos, tendo em vista o seu papel na produção científica brasileira, inclusive no que diz respeito ao ensino odontológico $^{10}$.

Avaliando a produção científica no país, é possível identificar que a distribuição geográfica do vínculo institucional dos pesquisadores é fortemente concentrada na região Sudeste, com destaque às capitais dos estados. A cidade de São Paulo, por exemplo, foi responsável por cerca de $20 \%$ da produção científica brasileira durante a última década ${ }^{11}$. Tais achados são corroborados pelos resultados da presente pesquisa, tendo em vista que $41,3 \%$ das publicações nacionais na Revista da ABENO se concentram no Sudeste do país, podendo tal heterogeneidade ser explicada pela maior concentração de cursos de graduação e pós-graduação nesta região ${ }^{9}$, que detém cerca de 43,6\% dos cursos de Odontologia ${ }^{12}$. Segundo o Ministério da Educação (2011) ${ }^{13}$, a distribuição das IES no território nacional, considerando apenas a localização de suas redes, mostra que $49 \%$ do total das instituições estão inseridas na região Sudeste. Porém, há tendência ao aumento da produção científica nas demais regiões, impulsionadas por ações governamentais que buscam expandir a oferta e a democratização do ensino superior, com o objetivo de diminuir a discrepância no território nacional. Observa-se a tendência de descentralização também neste estudo, que mostra crescente participação das regiões Norte, CentroOeste e, mais marcadamente, da região Nordeste ao longo da série histórica.

$\mathrm{O}$ aumento no número de profissionais formados, associado à expansão significativa dos cursos de graduação e pós-graduação lato e stricto sensu, tende a impulsionar o crescente desenvolvimento de pesquisas na área da Odontologia, sendo este último fator um importante responsável pelo aumento significativo na produção cientifica nacional em todas as áreas ${ }^{14,15}$.

Entre os temas mais abordados pela Revista da ABENO, quase metade dos artigos nesta série histórica tinham o processo de Ensino/ Aprendizagem em Odontologia como objeto de estudo. Destacaram-se também os temas referentes às Atividades de Estágio, Extensão e PET-Saúde e às Diretrizes Curriculares Nacionais, o que está de acordo com o perfil do periódico, o qual está diretamente voltado ao estudo do ensino odontológico.

Também foram frequentes os artigos abordando temas relacionados ao Mundo do Trabalho, retratando a expectativa e as perspectivas 
dos estudantes de graduação quanto à futura atuação profissional, além de estudos sobre a inserção profissional de egressos dos cursos de Odontologia. Da mesma forma, estudos de Epidemiologia e Serviços de Saúde foram frequentes. Nesta categoria, a maioria dos artigos se deteve ao estudo da prevalência de doenças e agravos à saúde entre estudantes e professores de Odontologia, do perfil dos pacientes atendidos em clínicas-escola e na avaliação da qualidade dos serviços de assistência odontológica ofertados pelas IES.

Ao longo dos seus 20 anos de existência, a Revista da ABENO tem desenvolvido papel fundamental na produção e divulgação do conhecimento científico relacionado às múltiplas facetas do processo de ensino-aprendizagem em Odontologia em âmbito nacional.

\section{CONCLUSÃO}

Ao longo de quase duas décadas, a Revista da ABENO apresentou volume crescente de publicações, cujos temas aderem ao escopo do periódico, com participação expressiva de IES, em especial das públicas, e com concentração desta produção na região Sudeste, embora com tendência ao aumento na participação das demais regiões geográficas.

\section{ABSTRACT \\ Bibliometric survey of articles published in} Revista da ABENO between 2001 and 2019

The present study aims to verify, through bibliometric analysis, the scientific publication profile of Revista da ABENO. The search strategy was conducted by two independent researchers on the journal's website, in which all the full published articles between 2001 and 2019 were analyzed. Upon reading the abstracts, the works were characterized according to the category of study, the theme addressed, year and volume of publication, in addition to data from the HEIs to which the authors of the article were linked, such as their status and geographical location. The researchers identified 486 articles published during the studied period. Mostly, the articles presented a methodological design of quantitative research (46.3\%), with the addressed themes related to the areas of Teaching / Learning in Dentistry (49.8\%); Internship, Extension and PET-Health Activities (15.2\%); and National Curricular Guidelines $(10.1 \%)$. The studies were developed and conducted mostly in public institutions $(70 \%)$, with a predominance of location in the Southeast region of Brazil (41.4\%). In conclusion, it appears that over nearly two decades Revista da ABENO has presented an increasing volume of scholarly articles, with a significant participation of tertiary education institutions, especially public ones, and with a concentration of this production in the Southeast region, although with a recent tendency to increase the participation of other geographic regions of the country

Descriptors: Bibliometrics. Dentistry.

Education, Dental.

\section{REFERÊNCIAS}

1. Araújo CA. Bibliometria: evolução histórica e questões atuais. Em Questão. 2006; 12(1)11-32.

2. Koo M. A bibliometric analysis of two decades of aromatherapy research. BMC Res Notes. 2017; 10:46.

3. Bhona FMC, Lourenço LM, Brum CRS. Violência doméstica: um estudo bibliométrico. Arq Bras de Psicol. 2011; 63(1):87-100.

4. Menezes JDV. Cláudio Ferreira de Melo: o arquiteto da ABENO. Fortaleza: Imprece Editorial, 2012. 80p.

5. Vanz SAS, Stumpf IRC. Procedimentos e ferramentas aplicados aos estudos bibliométricos. Inf \& Soc. 2010; 20(2)67-75.

6. Puerta AA, Faria LIL, Filho RCP. A importância dos estudos bibliométricos para o monitoramento de tecnologia: o caso na 
nanotecnologia no agronegócio. Encontro Brasileiro de Bibliometria e Cientometria. 2012; 3:1-7.

7. Site da revista da ABENO [homepage na internet]. Revista da ABENO agora é qualis B3. [acesso em 27 mar 2020]. Disponível em: revabeno.emnuvens.com.br/revabeno/ announcement/view/1.

8. Hilu L, Gisi ML. Produção científica no Brasil: um comparativo entre universidades públicas e privadas. Anais do $10^{\circ}$ Congresso Nacional de Educação. $1^{\circ}$ Seminário Internacional de Representações Sociais, Subjetividade e Educação; 2011 Nov 7-10; Curitiba: Champagnat; 2011. p. 5665-72.

9. Dutra DM, Freires IA, Neves RS, Lima DMB, Padilha WWN. Aspectos Institucionais e Metodológicos dos Resumos Brasileiros Publicados na $85^{\mathrm{a}}$ Reunião Anual Mundial da IADR - 2007. R Bras Ci Saúde. 2012; 16(4):545-52.

10. Barbosa LC, Saliba TA, Garbin CAS, Moimaz SAS. Panorama de pesquisas odontológicas brasileiras apresentadas em reunião científica - SBPqO. Rev Odontol. UNESP [online]. 2019; 48:e20190082.

11. Sidone OJG, Haddad EA, Mena-Chalco JP. A ciência nas regiões brasileiras: evolução da produção e das redes de colaboração científica. TransInformação. 2016; 28(1):15-31.

12. Martin ASS, Chisini LA, Martelli S, Sartori LRM, Ramos EC, Demarco FF. Distribuição dos cursos de Odontologia e de cirurgiõesdentistas no Brasil: uma visão do mercado de trabalho. Rev da ABENO. 2018; 18(1):6373.
13. Ministério da educação. Panorama e diagnóstico da oferta e qualidade da Educação Superior brasileira. Brasil: MEC; 2011. [Acesso em 27 de mar 2020]. Disponível em: portal.mec.gov.br/index. php?option=com_docman\&view=download $\underline{\text { \&alias }=13944 \text {-produto-1-senso-educ- }}$ superior-pdf\&Itemid=30192.

14. Gomes D, Agnoletto IG, Souza ML, Spiger V, Jakymiu JRG, Fuji EC, Mello ALSF. A produção científica da Odontologia e a Agenda Nacional de Prioridades de Pesquisa em Saúde. Rev ABENO. 2017; 17(2):11-21.

15. Cavalcante RA, Barbosa DR, Bonan PRF, Pires MBO, Martelli-Júnior H. Perfil dos pesquisadores da área de odontologia no Conselho Nacional de Desenvolvimento Científico e Tecnológico (CNPq). Rev Bras Epidemiol. 2008; 11(1):106-13.

\section{Correspondência para:}

Allan Vinícius Martins de Barros e-mail: allanmartinsodonto@gmail.com Hospital Universitário Oswaldo Cruz Universidade de Pernambuco Campus Santo Amaro.

Rua Arnóbio Marquês, 310, Santo Amaro, 50100-130 Recife/PE 